\title{
A NOTE ON UNIFORM ORDERED SPACES
}

\author{
HANS-PETER A. KÜNZI \\ (Received 15 July 1985 ; revised 3 January 1986) \\ Communicated by J. H. Rubinstein
}

\begin{abstract}
We characterize the generalized ordered topological spaces $X$ for which the uniformity $\mathscr{C}(X)$ is convex. Moreover, we show that a uniform ordered space for which every compatible convex uniformity is totally bounded, need not be pseudocompact.

1980 Mathematics subject classification (Amer. Math. Soc.): 06 B 30, 54 F 05.

We answer two questions of P. Fletcher and W. F. Lindgren. In [2, Problem F, page 94] they ask for necessary and sufficient conditions such that the uniformity $\mathscr{C}(X)$ of a $G O$ space $(X, \mathscr{T}, \leqslant)$ is convex with respect to $\leqslant$. In this note we show that the uniformity $\mathscr{C}(X)$ of a $G O$ spaces $X$ is convex if and only if each closed discrete subset of $X$ is countable. In [2, Problem I, p. 95] they ask whether a uniform ordered space $(X, \mathscr{T}, \leqslant)$ for which every convex uniformity compatible with $\mathscr{T}$ is totally bounded, is necessarily pseudocompact. They observe that the answer is positive, if $\leqslant$ is a linear order on $X$. In this note we show that the answer is negative in general. We will use the notation and the terminology of [2].
\end{abstract}

1.

In the first part of this note we will need the following lemma.

Lemma. Let $(X, \leqslant)$ be an uncountable linearly ordered set. Then there exists an uncountable subset $A$ of $X$ such that, if $a, b \in A$ and $a \leqslant b$, then there is $a$ $c \in X \backslash A$ with $a \leqslant c \leqslant b$.

(c) 1987 Australian Mathematical Society $0263-6115 / 87 \$ A 2.00+0.00$ 
Proof. Denote by $[X]^{3}$ the set of the subsets of $X$ with three elements. If $B \in[X]^{3}$, we denote the minimal element of $B$ by $L_{B}$ and the maximal element of $B$ by $R_{B}$. Finally, $M_{B}$ denotes the element of $B$ such that $L_{B}<M_{B}<R_{B}$.

Case 1. There exists a countable subset $D$ of $X$ so that for each $B \in[X]^{3}$ there is a $d \in D$ such that $L_{B} \leqslant d \leqslant R_{B}$. Set $C=X \backslash D$ and $A=\{x \in X \mid x$ is the smallest element of a convexity-component of $C$ in $X\}$. Since each convexitycomponent of $C$ in $X$ has at most two elements, $A$ is an uncountable set that satisfies the condition of the lemma.

Case 2. For each countable subset $D$ of $X$ there is a $B \in[X]^{3}$ such that $D \cap\left\{x \in X \mid L_{B} \leqslant x \leqslant R_{B}\right\}=\varnothing$. Define by transfinite induction for each $\beta<$ $\omega_{1}$ a set $B(\beta) \in[X]^{3}$ : Suppose that $B(\alpha)$ has been defined for each $\alpha<\beta$. There is a set $B \in[X]^{3}$ such that $\left\{x \in X \mid L_{B} \leqslant x \leqslant R_{B}\right\}$ contains no element of the countable set $\bigcup\{B(\alpha) \mid \alpha<\beta\}$. Set $B(\beta)=B$. Then $A=\left\{M_{B(\beta)} \mid \beta<\omega_{1}\right\}$ is uncountable and satisfies the condition of the lemma.

It is known that each $G O$ space is normal. In the next proof we will use the result that for a normal $T_{2}$-space $X$ the uniformity $\mathscr{C}(X)$ is the finest compatible uniformity on $X$ if and only if each locally finite open cover of $X$ has a countable open refinement of finite order [3, Remark following the proof of the theorem; compare 1 and 2, p. 190, \$5.28]. Recall that a topological space $X$ is called $\omega_{1}$-compact, if each closed discrete subset of $X$ is countable.

Proposition. Let $(X, \mathscr{T}, \leqslant)$ be a GO space. Then the following conditions are equivalent:

(a) $X$ is $\omega_{1}$-compact.

(b) $\mathscr{C}(X)$ is the finest uniformity for $(X, \mathscr{T})$.

(c) $\mathscr{C}(X)$ is convex.

Proof. (a) $\rightarrow$ (b). Since $X$ is $\omega_{1}$-compact and $\operatorname{dim} X \leqslant 1$, every locally finite open cover of $X$ is refined by a countable open refinement of finite order. We conclude that $\mathscr{C}(X)$ is the finest uniformity for $(X, \mathscr{T})$.

(b) $\rightarrow$ (c). Since the finest uniformity for a $G O$ space is convex [2, Theorem 4.33], $\mathscr{C}(X)$ is convex.

(c) $\rightarrow(a)$. Let $\mathscr{C}(X)$ be convex. Assume that $X$ is not $\omega_{1}$-compact. Then $X$ has an uncountable closed discrete subset $A$. By the lemma there exists an uncountable subset $A^{\prime}$ of $A$ such that every subset of $A^{\prime}$ that is convex in $A$ contains at most one point. Define a function $f: A \rightarrow R$ by $f(x)=0$ if $x \in A^{\prime}$, and $f(x)=1$ if $x \in A \backslash A^{\prime}$. Let $g: X \rightarrow R$ be continuous such that $g \mid A=f$. Let 
$V \in \mathscr{C}(X)$ such that $V \subset\left\{(x, y) \in X \times X:|g(x)-g(y)|<\frac{1}{2}\right\}$ and $V(x)$ is convex in $X$ for each $x \in X$. Since $V \in \mathscr{C}(X)$, there is a countable subset $D$ of $X$ such that $X=\bigcup\{V(d) \mid d \in D\}$. Clearly, each $V(d)$ contains at most one element of $A^{\prime}$ - a contradiction. We conclude that $X$ is $\omega_{1}$-compact.

EXAMPLE 1. Let $R$ denote the set of the reals and let $\leqslant$ denote the usual order on $R$. Consider the $G O$ space $(R, \mathscr{T}, \leqslant)$ where $\mathscr{T}$ denotes the discrete topology on $R$. Clearly $(R, \mathscr{T})$ is not $\omega_{1}$-compact. Hence $\mathscr{C}(R)$ is not convex.

\section{2.}

We construct a uniform ordered space $(X, \mathscr{T}, \leqslant)$ such that every convex uniformity compatible with $(X, \mathscr{T}, \leqslant)$ is totally bounded, but $X$ is not pseudocompact.

EXAMPLE 2. We use a modification of the well-known pseudocompact space $\psi$ [see 4, 5I]. Let $N$ be the set of the positive integers and let $\Gamma$ be an infinite maximal almost disjoint family of infinite subsets of $N$. As usual set $\psi=N \cup \Gamma$. Let $\left(a_{2 n-1}\right)_{n \in N}$ be a sequence of pairwise different elements of $\Gamma$. Set $a_{2 n}=-n$ for each $n \in N, A=\left\{a_{2 n} \mid n \in N\right\}$, and $D=\left\{a_{n} \mid n \in N\right\}$. Let $X=N \cup \Gamma \cup A$. Consider the following collection of subsets of $X$ :

$$
\begin{aligned}
& \mathscr{B}=\{\{n\} \mid n \in N\} \cup\{(E \backslash F) \cup\{E\} \mid E \in \Gamma \backslash D, F \text { is a } \\
& \text { finite subset of } N\} \cup\left\{\cup_{n=1}^{k} G\left(a_{n}\right) \mid k \in N ; G\left(a_{2 m}\right)=\left\{a_{2 m}\right\}\right. \\
& \text { (for each } m \in N \text { such that } 2 m \leqslant k) ; G\left(a_{2 m-1}\right)=\left(a_{2 m-1} \backslash\right. \\
& F) \cup\left\{a_{2 m-1}\right\} \text { where } F \text { is a finite subset of } N \text { (for each } \\
& m \in N \text { such that } 2 m-1 \leqslant k)\} .
\end{aligned}
$$

Set $\mathscr{S}=\{[X \times G] \cup[(X \backslash G) \times X] \mid G \in \mathscr{B}\}$. Then $\mathscr{S}$ is a subbase for a quasiuniformity $\mathscr{U}$ on $X$. Consider the topology $\mathscr{T}\left(\mathscr{U}^{*}\right)$ on $X$ where $\mathscr{U}^{*}$ denotes the uniformity generated by $\left\{V \cap V^{-1} \mid V \in \mathscr{U}\right\}$ on $X$. One easily checks that the points of the $\mathscr{T}\left(\mathscr{U}^{*}\right)$-open subspace $\psi$ of $X$ have their usual neighbourhoods. Moreover, each point of $X \backslash \psi$ is isolated in $X$. Hence $\mathscr{T}\left(\mathscr{U}^{*}\right)$ is not pseudocompact. Since $\mathscr{T}\left(\mathscr{U}^{*}\right)$ is a Hausdorff topology, $\left(X, \mathscr{T}\left(\mathscr{U}^{*}\right), \cap \mathscr{U}\right)$ is a uniform ordered space [2, pages 81,84$]$. Let $\mathscr{W}$ be a convex uniformity compatible with $\left(X, \mathscr{T}\left(\mathscr{U}^{*}\right), \cap \mathscr{U}\right)$. Let $Z \in \mathscr{W}$. Since $\mathscr{W}$ is convex, there is a $Z^{\prime} \in \mathscr{W}$ such that $Z^{\prime} \subset Z$ and $Z^{\prime}(x)$ is convex in $X$ for each $x \in X$. Since $\psi$ is a pseudocompact subspace of $X$, there is a finite subset $F$ of $\psi$ such that $\psi \subset Z^{\prime}(F)$. Hence there is an $x \in F$ such that $Z^{\prime}(x)$ contains infinitely many points of $D \backslash A$. Note that, if 
$k, n \in N$ and $k \leqslant n$, then $\left(a_{n}, a_{k}\right) \in \cap \mathscr{U}$. Since $Z^{\prime}(x)$ is convex in $X, Z^{\prime}(x)$ contains all but finitely many points of $D$. We conclude that $\mathscr{W}$ is totally bounded.

\section{References}

[1] H. H. Corson and J. R. Isbell, 'Euclidean covers of topological spaces', Quart. J. Math. Oxford Ser. (2) 11 (1960), 34-42.

[2] P. Fletcher and W. F. Lindgren, Quasi-uniform spaces (Lecture Notes in Math., Dekker, New York, 1982).

[3] P. Fletcher, H. Junnila and W. Lindgren, 'Another note on bounded paracompactness', manuscript.

[4] L. Gillman and M. Jerison, Rings of continuous functions (Van Nostrand, Princeton, N. J., $1960)$.

Department of Mathematics

University of Bern

Sidlerstr. 5, 3012 Bern

Switzerland 